\title{
Deconstructing endothelial dysfunction: soluble guanylyl cyclase oxidation and the NO resistance syndrome
}

\author{
Mark T. Gladwin
}

Vascular Medicine Branch, National Heart, Lung, and Blood Institute, and Critical Care Medicine Department, Clinical Center, National Institutes of Health, Bethesda, Maryland, USA.

\begin{abstract}
In this issue of the JCI, Stasch and colleagues suggest that a novel drug, BAY 58-2667, potently activates a pool of oxidized and heme-free soluble guanylyl cyclase (sGC; see the related article beginning on page 2552). The increased vasodilatory potency of BAY 58-2667 the authors found in a number of animal models of endothelial dysfunction and in human blood vessels from patients with diabetes suggests that there exists a subphenotype of endothelial dysfunction characterized by receptor-level NO resistance. Diseases associated with NO resistance would appear to be ideally suited for therapies directed at restoring redox homeostasis, sGC activity, and NO sensitivity.
\end{abstract}

Our molecular understanding of the pathogenesis of diabetes mellitus crystallized with the discovery of insulin and the catastrophic failure to produce insulin in type 1 disease. It would take decades to unravel the mechanisms underlying type 2 diabetes, a more common disease associated with preserved insulin production but resistance to insulin at the receptor level. A similar march to discovery characterizes most endocrinopathies; e.g., the identification of a failure to produce thyroid hormone in hypothyroidism and later the discovery of the more unusual generalized resistance to thyroid hormone. If we consider the diatomic free radical NO, a paracrine and endocrine signaling molecule (1), we should not be surprised by the ultimate discovery of "NO resistance syndromes."

Endothelial NO is produced by the endothelial isoform of NOS, eNOS, via a 5-electron oxidation of $\mathrm{L}$-arginine to form L-citrulline and NO. eNOS is activated following stimulation with calcium ionophore, muscarinic receptor activation by acetylcholine, delivery of excess substrate arginine, and shear stress. NO then diffuses as a paracrine signaling molecule

Nonstandard abbreviations used: BAY 58-2667, 4-[((4-carboxybutyl) \{2-[(4-phenethylbenzyl)oxy] phenethyl\}amino) methyl [benzoic]acid; cGMP, cyclic GMP; DEA/NO, donor 2-( $N, N$-diethylamino)-diazenolate-2-oxide; sGC, soluble guanylyl cyclase.

Conflict of interest: The author has declared that no conflict of interest exists.

Citation for this article: J. Clin. Invest. 116:2330-2332 (2006). doi:10.1172/JCI29807. to albuminal smooth muscle and binds to the hemes on the $\alpha / \beta$ heterodimer soluble guanylyl cyclase (sGC), which in turn converts GTP to cyclic GMP (cGMP) and activates cGMP-dependent protein kinases (Figure 1) (2-4). On the heels of the discovery of this pathway, it became clear that patients with coronary artery disease or its risk factors - diabetes, hypercholesterolemia, hypertension, atherosclerosis, increasing age, and tobacco smoking - develop endothelial dysfunction. The observed impairments in stimulated and basal NO production are now classic: The expected blood flow responses to infusion with acetylcholine, an endothelium-dependent vasodilator, are reduced, and the normal decrease in blood flow during NGmonomethyl L-arginine infusion, a direct NOS inhibitor, are blunted (5). However, it is important to note that in all of these pathologies the vasodilatory responses to endothelium-independent exogenous NO, typically assessed by the infusion of sodium nitroprusside, are preserved.

This relatively simple signaling paradigm becomes increasingly complex as we begin to consider factors that modulate substrate transport and availability for NOS, phosphorylation, and posttranslational modifications of NOS; oxidative uncoupling of the enzyme; and downstream stability of the secondary messenger cGMP. Indeed, the enzymes arginase I and II can degrade arginine, and oxidative stress can uncouple eNOS, leading to a state of L-arginine resistance (6-8). Once again, we must note that in all of these pathologies the vasodilatory responses to authentic $\mathrm{NO}$ are generally preserved.

\section{Is there a subphenotype of endothelial dysfunction characterized by NO resistance?}

In this issue of the JCI, Stasch and colleagues present provocative findings that clinical states of endothelial dysfunction can be associated with the accumulation of oxidized and heme-free sGC that cannot be activated by NO (9). Indeed, the oxidation of purified sGC enzyme, endothelial cells, platelets, or aortic ring bioassay preparations with $1 \mathrm{H}-[1,2,4]$ oxadiazolo [3,4-a]quinoxalin-1-one (ODQ) or peroxynitrite produces a state of NO resistance in which both NO-dependent cGMP accumulation and vasodilation are impaired. Remarkably, the authors provide extensive and compelling experimental evidence that the NO- and heme-independent activator of sGC, 4-[((4-carboxybutyl) \{2-[(4phenethylbenzyl)oxy] phenethyl (amino) methyl [benzoic]acid (BAY 58-2667), can potently bind to and activate these oxidized and/or heme-free sGCs, producing selective sGC activation and vasodilation of diseased blood vessels (Figure 1). This binding also appears to inhibit ubiquitindependent sGC protein degradation. The thesis that oxidized and heme-free sGC contributes to endothelial dysfunction in clinical conditions is further supported by in vivo and vascular ring experiments in the spontaneously hypertensive rat, Watanabe hyperlipidemic rabbits, and $\mathrm{ApoE}^{-/-}$mice on a high-fat diet as well as in isolated human mesocolon arteries from patients with type 2 diabetes.

While these studies suggest that atherosclerosis and its risk factors would be associated with impaired sGC function and resistance to exogenous NO, we know that patients with endothelial dysfunction have preserved responses to nitroprusside 


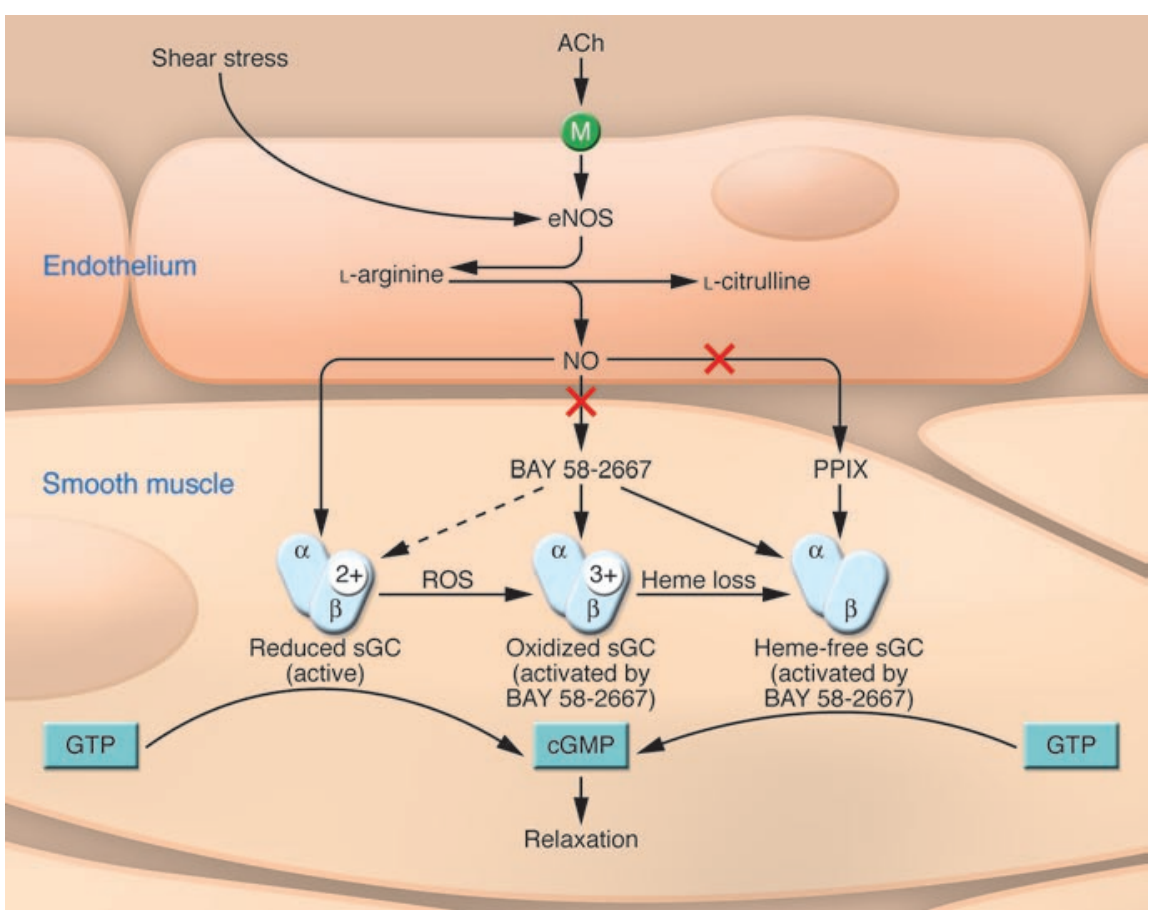

and nitroglycerin. As described above, the sine qua non of endothelial dysfunction is impaired vasodilatory response to acetylcholine but normal response to sodium nitroprusside or nitroglycerine. In fact, in the current study by Stasch et al. (9), while responses to donor 2-(N,N-diethylamino)diazenolate-2-oxide (DEANO; an NO donor) were blunted after treatment with peroxynitrite, the blood flow responses to sodium nitroprusside were similar in the Watanabe hyperlipidemic rabbits to those of the control New Zealand rabbits, and there was little difference in nitroglycerindependent vasodilation between $\mathrm{ApoE}^{-/-}$ mice on high- and low-fat diets. These models indicate a more profound effect of BAY 58-2667 in the ApoE-/- mice and Watanabe rabbits than the severity of resistance to NO. In order to reconcile these results we must consider 3 issues relevant to the subject of NO resistance.

One possibility is that the increased potency of BAY 58-2667 amplifies the cGMP production from a small subpopulation of oxidized or heme-free sGCs. Indeed, the $50 \%$ effective concentration for DEANO, nitroglycerin, and sodium nitroprusside all approximate $1 \times 10^{-7} \mathrm{~g} / \mathrm{ml}$, while BAY 58-2667 ranges from $1 \times 10^{-9} \mathrm{~g} / \mathrm{ml}$ to $1 \times 10^{-10} \mathrm{~g} / \mathrm{ml}$ for normal and oxidized vessels, respectively. If true, this would suggest that intra-arterial infusions of BAY 58-2667 should be added to our classic tests of endothelial dysfunction to quantify the severity of sGC oxidation and $\mathrm{NO}$ resistance.

A second possibility is that we have failed to completely deconstruct endothelial dysfunction subphenotypes. Is it possible that buried within the error of the mean of blood flow responses to NO donors are subpopulations of patients with blunted responses to nitroglycerin and sodium nitroprusside? Consistent with this possibility, in sickle cell disease and other hemolytic conditions, intravascular hemolysis releases hemoglobin into plasma, which reacts with and inactivates $\mathrm{NO}$, producing a state of NO resistance (10-12). These hemolytic diseases are also associated with significant oxidant stress, which would oxidize sGC (13). While results of an examination of a group of sickle cell disease patients, regardless of clinical severity, suggests that the blood flow responses to sodium nitroprusside are normal, a subgroup analysis of male patients and those with a high degree of intravascular hemolysis revealed a state of NO resistance $(10,14)$. Indeed, the patients with sickle cell disease with high-level hemolysis and the transgenic mouse models of sickle cell disease exhibit profound resistance to infusions of nitroglycerin, sodium nitroprusside, and the direct NO donors, the NONOates (like DEANO and DETANO; refs. 10, 15-18). Clearly, more study is required to determine

\section{Figure 1}

Oxidized and heme-free SGC is unresponsive to NO but activated by BAY 58-2667. Upon activation by shear stress or acetylcholine (ACh), endothelial NO is produced by eNOS. $\mathrm{NO}$ then diffuses to smooth muscle and binds to the reduced hemes $\left(\mathrm{Fe}^{2+}\right.$ ferrous heme) on the $\alpha / \beta$ heterodimer sGC, which in turn converts GTP to cGMP and leads to downstream smooth muscle relaxation and vasodilation. Clinical states of endothelial dysfunction can be associated with the accumulation of oxidized ( $\mathrm{Fe}^{3+}$ ferric heme) and heme-free sGC that cannot be activated by NO. A study in this issue of the $\mathrm{JCl}$ demonstrates that the NO-and hemeindependent activator of SGC, BAY 58-2667, can potently bind to and activate the oxidized and heme-free sGC, producing vasodilation of diseased blood vessels, while the porphyrin ligand protoporphyrin IX (PPIX) only binds to heme-free SGC (9). M, muscarinic receptor.

whether these conditions reflect an unusual form of endothelial dysfunction or whether subgroups of patients with endothelial dysfunction exhibit blunted responses to nitroglycerin or sodium nitroprusside.

A final, more confusing issue must be addressed. Not all NO-dependent vasodilations and not all NO donors are the same. This was recently highlighted by a provocative study by Adachi and colleagues, which suggested that NO-dependent vasodilation occurs via the intermediate formation of peroxynitrite, which in turn oxidizes and S-glutathionylates a reactive thiol on sarcoplasmic reticulum $\mathrm{Ca}^{2+}$ ATPase (SERCA; ref. 19). In this study, scavengers of superoxide and peroxynitrite inhibited vasodilation of aortic rings to acetylcholine and authentic NO (in solution), while responses to sodium nitroprusside were normal. The authors speculated that nitroprusside directly activated sGC, while authentic NO reacted with superoxide to form peroxynitrite and oxidize SERCA. Regardless of the specific mechanism, this study highlights the fact that within an experimental system, the vasodilatory responses to sodium nitroprusside can be normal even as the responses to authentic NO are impaired.

Indeed, sodium nitroprusside and nitroglycerin, which are both used clinically to test endothelial responses to exogenous NO, are irregular NO donors. Nitroprusside is "dirty" in that it can release iron and cyanide, which 
in turn produces oxidant stress. Furthermore, nitroprusside actually generates $\mathrm{NO}^{+}$, the nitrosonium cation, which in addition to reducing $\mathrm{NO}$ can form nitrite and S-nitrosothiols, both physiologically active vasodilators in their own right $(20,21)$. Nitroglycerin must be enzymatically converted to $\mathrm{NO}$ and nitrite, and the role of $\mathrm{NO}$ formation in the mechanism of nitroglycerin-dependent vasodilation is debatable. Sustained exposure to nitroglycerin (nitrates) results in pharmacological tolerance or resistance within days; the mechanisms of tolerance are incompletely understood, but a contributing role of increased oxidant stress and superoxide production has been proposed (22). It is intriguing to speculate that sGC oxidation might contribute, at least in part, to nitrate tolerance. All of these issues highlight the remarkable uncertainty about the chemistry and mechanisms underlying our most basic tools used to test endothelial function and even the precise mechanisms of "NO-dependent" vasodilation.

In conclusion, the increased biological activity of ligands that potently activate a pool of oxidized and heme-free sGC suggests that there exists a state of receptorlevel NO resistance. In order to understand whether NO resistance represents a real subphenotype of endothelial dysfunction, more work is required to characterize the capacity of blood vessels in such patients to respond to drugs like BAY 58-2667 and to a range of NO donors. Patients with enhanced vasodilatory responses to BAY 58-2667 but blunted vasodilator responses to NO donors would appear to be ideally suited for thera- pies directed at restoring redox homeostasis, sGC activity, and NO sensitivity.

Address correspondence to: Mark T. Gladwin, Vascular Medicine Branch, National Heart, Lung, and Blood Institute, Critical Care Medicine Department, Clinical Center, National Institutes of Health, Building 10-CRC, Room 5-5140, 10 Center Drive, Bethesda, Maryland 20892-1662, USA. Phone: (301) 435-2310; Fax: (301) 451-7091; E-mail:mgladwin@nih.gov.

1. Schechter, A.N., and Gladwin, M.T. 2003. Hemoglobin and the paracrine and endocrine functions of nitric oxide. N. Engl. J. Med. 348:1483-1485.

2. Furchgott, R.F., and Zawadzki, J.V. 1980. The obligatory role of endothelial cells in the relaxation of arterial smooth muscle by acetylcholine. Nature. 288:373-376

3. Ignarro, L.J., Byrns, R.E., Buga, G.M., and Wood, K.S. 1987. Endothelium-derived relaxing factor from pulmonary artery and vein possesses pharmacologic and chemical properties identical to those of nitric oxide radical. Circ. Res. 61:866-879.

4. Palmer, R.M., Ferrige, A.G., and Moncada, S. 1987. Nitric oxide release accounts for the biological activity of endothelium-derived relaxing factor. Nature. 327:524-526.

5. Quyyumi, A.A., et al. 1995. Nitric oxide activity in the human coronary circulation. Impact of risk factors for coronary atherosclerosis. J. Clin. Invest. 95:1747-1755.

6. Wu, G., and Morris, S.M., Jr. 1998. Arginine metabolism: nitric oxide and beyond. Biochem J. 336:1-17.

7. Berkowitz, D.E., et al. 2003. Arginase reciprocally regulates nitric oxide synthase activity and contributes to endothelial dysfunction in aging blood vessels. Circulation. 108:2000-2006.

8. Elrod, J.W., et al. 2006. eNOS gene therapy exacerbates hepatic ischemia-reperfusion injury in diabetes: a role for eNOS uncoupling. Circ. Res. 99:78-85.

9. Stasch, J.-P., et al. 2006. Targeting the heme-oxidized nitric oxide receptor for selective vasodilatation of diseased blood vessels. J. Clin. Invest.
116:2552-2561. doi:10.1172/JCI28371.

10. Reiter, C.D., et al. 2002. Cell-free hemoglobin limits nitric oxide bioavailability in sickle-cell disease. Nat. Med. 8:1383-1389.

11. Minneci, P.C., et al. 2005. Hemolysis-associated endothelial dysfunction mediated by accelerated NO inactivation by decompartmentalized oxyhemoglobin. J. Clin. Invest. 115:3409-3417. doi:10.1172/JCI25040.

12. Morris, C.R., et al. 2005. Dysregulated arginine metabolism, hemolysis-associated pulmonary hypertension, and mortality in sickle cell disease. JAMA. 294:81-90.

13. Aslan, M., et al. 2001. Oxygen radical inhibition of nitric oxide-dependent vascular function in sickle cell disease. Proc. Natl. Acad. Sci. U. S. A. 98:15215-15220.

14. Gladwin, M.T., et al. 2003. Divergent nitric oxide bioavailability in men and women with sickle cell disease. Circulation. 107:271-278.

15. Nath, K.A., et al. 2000. Mechanisms of vascular instability in a transgenic mouse model of sickle cell disease. Am. J. Physiol. Regul. Integr. Comp. Physiol. 279:R1949-R1955.

16. Kaul, D.K., Liu, X.D., Fabry, M.E., and Nagel, R.L. 2000. Impaired nitric oxide-mediated vasodilation in transgenic sickle mouse. Am. J. Physiol. Heart Circ. Physiol. 278:H1799-H1806.

17. Kaul, D.K., Liu, X.D., Chang, H.Y., Nagel, R.L., and Fabry, M.E. 2004. Effect of fetal hemoglobin on microvascular regulation in sickle transgenicknockout mice. J. Clin. Invest. 114:1136-1145. doi:10.1172/JCI200421633.

18. Eberhardt, R.T., et al. 2003. Sickle cell anemia is associated with reduced nitric oxide bioactivity in peripheral conduit and resistance vessels. Am. J. Hematol. 74:104-111.

19. Adachi, T., et al. 2004. S-Glutathiolation by peroxynitrite activates SERCA during arterial relaxation by nitric oxide. Nat. Med. 10:1200-1207.

20. Stamler, J.S., et al. 1992. S-nitrosylation of proteins with nitric oxide: synthesis and characterization of biologically active compounds. Proc. Natl. Acad. Sci. U. S. A. 89:444-448.

21. Cosby, K., et al. 2003. Nitrite reduction to nitric oxide by deoxyhemoglobin vasodilates the human circulation. Nat. Med. 9:1498-1505.

22. Fayers, K.E., Cummings, M.H., Shaw, K.M., and Laight, D.W. 2003. Nitrate tolerance and the links with endothelial dysfunction and oxidative stress. Br. J. Clin. Pharmacol. 56:620-628. 